\title{
Isolation and Identification of ER Associated Proteins with Unique Expression Changes Specific to the V144D SPTLC1 Mutations in HSN-I
}

\section{Scott E Stimpson ${ }^{1,3,4}$, Antonio Lauto ${ }^{4,6}$, Jens R Coorssen ${ }^{2,3,4,5 *}$ and Simon J Myers ${ }^{1,3,4,5 *}$}

${ }^{1}$ Neuro-Cell Biology Laboratory, Western Sydney University, Australia

${ }^{2}$ Molecular Physiology, Western Sydney University, Australia

${ }^{3}$ Molecular Medicine Research Group, Western Sydney University, Australia

${ }^{4}$ School of Science and Health, Western Sydney University, Australia

${ }^{5}$ School of Medicine, Western Sydney University, Australia

${ }^{6}$ Biomedical Engineering and Neuroscience (BENS), Western Sydney University, Australia

\begin{abstract}
Axonal degeneration is the final common path in many neurological disorders. Hereditary sensory neuropathies (HSN) are a group of neuropathies involving the sensory neurons. The most common subtype is autosomal dominant hereditary sensory neuropathy type I (HSN-I). Progressive degeneration of the dorsal root ganglion (DRG) neuron with an onset of clinical symptoms between the second or third decade of life characterises HSN-I. Mutations in the serine palmitoyltransferase (SPT) long chain subunit 1 (SPTLC1) gene cause HSN-I. The endoplasmic reticulum (ER) is a dynamic organelle that houses the SPTLC1 protein. Ultra structural analysis has shown the ER in the HSN-I mutant cells to wrap around dysfunctional mitochondria and tethers them to the perinucleus.

This investigation establishes that the V144D mutant of SPTLC1 alters the expression of and potentially interacts with a set of proteins within the ER. Using ER protein lysates from HSN-I patient and control lymphoblasts: we have identified a change in regulation of five proteins; Hypoxia Up regulated Protein 1: Chloride intracellular channel protein 1: Ubiqutin-40s Ribosomal protein S27a: Coactosin and Ig Kappa chain C. The expression and regulation of these proteins may help to establish a link between the ER and the 'dying back' process of the DRG neuron.
\end{abstract}

Keywords: Hereditary sensory neuropathy type 1; Serine palmitoyltransferase long chain sub-unit 1; Endoplasmic reticulum; Oxidative stress

Abbreviations: HSN: Hereditary Sensory Neuropathies; HSN-I: Hereditary Sensory Neuropathy Type I; DRG: Dorsal Root ganglion; SPT: Serine palmitoyl transferase; SPTLC1: Serine palmitoyl transferase long chain subunit 1; ER: Endoplasmic Reticulum; LCB1: LongChain Base One; UPR: Unfolded Protein Response; UPS: Ubiquitin Proteasome System; ROS: Reactive Oxygen Species; ORP-150: Hypoxia up Regulated Protein 1; CLIC1: Chloride Intracellular Channel Protein 1; RPS27a: Ubiqutin-40s Ribosomal Protein s27a; COTL1: Coactosin; HRP: Horse Radish Peroxidase; IEF: Isoelectric Focusing; TEMED: Tetramethylethylenediamine; PMT: Photomultiplier Tube; 2DE: Two Dimensional Gel Electrophoresis; IAA: Idoacetic acid; LC/ MS: Liquid Chromatography/Mass Spectrometry; ECL: Enhanced Chemiluminescence; FACS: Fluorescence's Assisted Cell Sorting; Grp170: Glucose related protein 170; G-actin: Globular actin; F-actin: Filamentous actin

\section{Introduction}

The ER is an intracellular organelle which supports and maintains a plethora of functions critical for cellular survival. The ER plays a crucial role in many aspects of protein compartmentalisation which include membrane translocation, folding, post-translational modification, and transport of both membrane and soluble proteins [1]. In addition, the ER is involved in the synthesis of phospholipids and steroids and also in the regulation of $\mathrm{Ca}^{2+}$ homeostasis. The ER is organised in a complex: continuous network of tubules and sheets that includes the nuclear envelope and extends throughout the cytosol into the cell periphery [1]. Nascent polypeptide chains fold, acquire further modifications like glycosylation and disulphide bonds and often assemble with other subunits before traversing further along the secretory pathway with in the ER. These processes are both assisted and monitored by molecular chaperones [1].

SPT is an ER bound and key rate determining enzyme in the complex sphingolipid metabolic pathway consisting of 3 subunits; SPTLC1: SPTLC2 and SPTLC3 [2]. Mutations within SPTLC1 result in potential dysfunction and perturbations in sphingolipid synthesis and metabolism causing HSN-I [2]. These mutations are single amino acid changes in the SPTLC1 gene that encodes the long-chain base one (LCB1) subunit [3]. HSN-I is the most common subtype of HSN [4]. HSN-I is an autosomal dominant disorder characterised by degeneration of the DRG neuron and with a clinical onset between the second or third decades of life.

The ER carries out extensive quality control of proteins to enable normal cellular function. Disruption to the function of the ER or loss of its integrity leads to ER stress [5]. ER stress can be characterised by the accumulation of unfolded proteins and changes in calcium homeostasis within the ER with stress activating the unfolded protein response (UPR) [6]. The UPR and its signalling components can change the expression of specific proteins such as, those designated for the ER chaperones; the enhancement of degradation of misfolded (mutant or unfolded) proteins; and the inhibition of protein synthesis to decrease the load within the ER [6].

*Corresponding author: Simon J. Myers, Western Sydney University, Campbelltown campus, Locked Bag 1797, Penrith, Australia, Tel: +61 024620 3383; E-mail: s.myers@uws.edu.au

Jens Coorssen, Professor, Western Sydney University, Campbelltown campus, Locked Bag 1797, Penrith, Australia, Tel: +61 4620 3802; E-mail: j.coorssen@uws.edu.au

Received: February 06, 2016; Accepted: February 15, 2016; Published February 18, 2016

Citation: Stimpson SE, Lauto A, Coorssen JR, Myers SJ (2016) Isolation and Identification of ER Associated Proteins with Unique Expression Changes Specific to the V144D SPTLC1 Mutations in HSN-I. Biochem Anal Biochem 5: 248. doi:10.4172/2161-1009.1000248

Copyright: (c) 2016 Stimpson SE, et al. This is an open-access article distributed under the terms of the Creative Commons Attribution License, which permits unrestricted use, distribution, and reproduction in any medium, provided the original author and source are credited. 
Disturbed functions of the ubiquitin proteasome system (UPS): responsible for the degradation of cytosolic: ER and synaptic proteins: can contribute to ER stress [5]. A common occurrence in many neurodegenerative disorders is the accumulation and deposits of misfolded proteins that affects various cell signalling systems: as well as neuronal connectivity and cell death such as in Alzheimer's: Parkinson's and Huntington's disorders.

The activity of the UPS is mitigated in degenerative disorders by the protein aggregation or by enhanced oxidative stress with other toxic products [5]. Dysfunctional UPS in turn causes increased accumulation of proteins in the cell leading to ER stress and the aggravation of the disorder [5]. Additionally the effect of environmental toxins, reactive oxygen species (ROS) and other signals that influence mitochondria lead to the activation of the caspase family of cysteine proteases causing cell death [7]. This negative cycle of increasing 'stressors' within the cell often leads to the cells inability to function properly and eventually leading to cell death [7].

It has recently been shown that HSN-I patient cells, expressing the V144D SPTLC1 mutant have a marked increase in ER stress in comparison to healthy control cells [8]. Considering this finding: in this study we have investigated altered protein changes in the ER membrane of these HSN-I patient cells. The resulting proteomic and cell biology analyses have identified for the first time, increases in the Hypoxia up regulated Protein 1 (ORP-150), Chloride intracellular channel protein 1 (CLIC1), Ubiqutin-40s ribosomal protein S27a (RPS27a), Coactosin (COTL1) and Ig Kappa chain C protein expression in HSN-I in this membrane.

\section{Materials and Methods}

All cell culture stock solutions, including RPMI-1640, Foetal bovine serum (FBS), Penicillin $(100 \mathrm{U} / \mathrm{mL})$, Streptomycin $(100 \mu \mathrm{g} / \mathrm{mL})$, L-glutamine $(2 \mathrm{mM})$, HEPES $(1 \mathrm{M})$ : and phosphate buffered saline (PBS) were purchased from GIBCO Invitrogen (Australia). Cell culture consumables were purchased from BD Falcon (Greiner, USA). DAPI stains were purchased from Sigma-Aldrich (Australia).

\section{EBV Transformed lymphoblasts}

EBV transformed control and V144D HSN-I patient lymphoblasts were kindly provided by Prof. Garth Nicholson (Molecular Medicine Laboratory: Anzac Research Institute: Sydney) [9].

\section{Lymphoblast cultures}

Lymphoblasts were cultured in RPMI-1640 media (GIBCO), supplemented with FBS $(10 \% \mathrm{v} / \mathrm{v})$, Penicillin $(1 \mathrm{U} / \mathrm{mL})$, Streptomycin $(1 \mu \mathrm{g} / \mathrm{mL})$, L-glutamine $(2 \mathrm{mM})$, and HEPES $(1 \mathrm{M})$ at $37^{\circ} \mathrm{C}$ in a humidified atmosphere of $5 \% \mathrm{CO}_{2}$, using $\mathrm{T} 75 \mathrm{~cm}^{2}$ culture flasks (Greiner: Interpath). Prior to use in biochemical assays, lymphoblasts were collected by centrifugation at $1,500 \times g(5 \mathrm{~min}$ at RT $)$ and washed in PBS. Cell counts were obtained using the Countess Automated Cell Counter (Invitrogen, Australia).

\section{Isolation of ER proteins}

Briefly, ER proteins were isolated using a sucrose density gradient $[10,11]$. Lymphoblasts were first centrifuged at $1,500 \times g$ for $5 \mathrm{~min}$, and the cells were then washed in $10 \mathrm{~mL}$ of ice cold $1 \times \mathrm{PBS}$ prior to suspension in $10 \mathrm{~mL}$ ice cold CaSRB Buffer $(10 \mathrm{mM} \mathrm{NaCl}, 1.5 \mathrm{mM}$ $\mathrm{CaCl}, 10 \mathrm{mM}$ Tris-HCL, pH 7.5) and left on ice for $10 \mathrm{~min}$. Cells were homogenised using a Dounce homogenizer (Kimble-Chase, USA) and $7 \mathrm{~mL}$ of $2.5 \times$ MS buffer $(210 \mathrm{mM}$ Mannitol, $70 \mathrm{mM}$ sucrose, 5
mM EDTA, $5 \mathrm{mM}$ Tris- $\mathrm{HCl}, \mathrm{pH}$ 7.6) was added to restore isotonicity. Homogenate was centrifuged at $700 \mathrm{x} g$ for $5 \mathrm{~min}$ to remove nuclei and unbroken cells. The resulting supernatant was centrifuged at $15,000 \mathrm{x} g$ for $10 \mathrm{mins}$ to remove mitochondria. A sucrose gradient was made in $15 \mathrm{~mL}$ high speed centrifuge tubes (Beckman Coulter, USA) by adding $2 \mathrm{~mL}$ of $2.0 \mathrm{M}$ sucrose buffer (2.0 M sucrose, $10 \mathrm{mM}$ Tris-base, $0.1 \mathrm{mM}$ EDTA, pH 7.6) overlayed with $3.0 \mathrm{~mL}$ of $1.5 \mathrm{M}$ sucrose buffer $(1.5 \mathrm{M}$ sucrose, $10 \mathrm{mM}$ Tris-base, $0.1 \mathrm{mM}$ EDTA, $\mathrm{pH} 7.6$ ) and $3.0 \mathrm{~mL}$ of 1.3 $\mathrm{M}$ sucrose (1.3 $\mathrm{M}$ sucrose, $10 \mathrm{mM}$ Tris-base, $0.1 \mathrm{mM}$ EDTA, $\mathrm{pH}$ 7.6) ER containing supernatant was loaded on top of the sucrose gradient and spun at 152,000 $\times \mathrm{g}$ for $70 \mathrm{~min}$. The ER band, the interface of the $1.5 \mathrm{M}$ and $1.3 \mathrm{M}$ sucrose, was gently removed using a $20 \mathrm{G}$ needle, transferred to a $4 \mathrm{~mL}$ high speed centrifuge tubes (Beckman Coulter, USA) and centrifuged at $100,000 \times \mathrm{g}$ for $35 \mathrm{~min}$. The resulting pellet was resuspended in 2D solubilisation buffer containing $8 \mathrm{M}$ urea, $2 \mathrm{M}$ thiourea, $4 \%(\mathrm{w} / \mathrm{v})$ CHAPS and a cocktail of protease inhibitors.

\section{Protein concentration}

Determination of total cellular protein was performed using the EZQ protein estimation assay (Invitrogen, Australia) as previously described by Churchward [12].

\section{Two dimensional gel electrophoresis}

Protein concentration estimations (EZQ assay) were performed on patient and control ER protein fractions; a total of $100 \mu \mathrm{g}$ protein was used for each 2DE analysis. 2DE was carried out as previously described [13-16]. Briefly, ER proteins were reduced and alkylated in solutions containing total protein extraction buffer (containing $8 \mathrm{M}$ urea, $2 \mathrm{M}$ thiourea and 4\% CHAPS without ampholytes), total extraction buffer with $2 \%$ ampholytes, TBP/DTT disulphide reduction buffer $(2.3 \mathrm{mM}$ Tributyl phosphine and $45 \mathrm{mM}$ DTT) and alkylation buffer $(230 \mathrm{mM}$ acrylamide monomer).

The treated samples were added to $7 \mathrm{~cm}$ Non-Linear $\mathrm{pH}$ 3-10 IPG strips (Bio-Rad ReadyStrip), and rehydrated for $16 \mathrm{hrs}$ at RT. Isoelectric focusing (IEF) was then carried out at $20^{\circ} \mathrm{C}$ using the Protean IEF Cell (Bio-Rad, USA). After IEF, IPG strips were then resolved in the second dimension using a $12.5 \% \mathrm{~T}, 2.6 \% \mathrm{C}$ polyacrylamide gel buffered with $375 \mathrm{mM}$ Tris buffer ( $\mathrm{pH} 8.8$ ), $0.1 \%(\mathrm{w} / \mathrm{v})$ sodium dodecyl sulphate and polymerised with $0.05 \%(\mathrm{w} / \mathrm{v})$ ammonium persulphate and $0.05 \%(\mathrm{v} / \mathrm{v})$ tetramethylethylenediamine (TEMED). A stacking gel containing a 5\% T, 2.6\% C polyacrylamide buffered with $375 \mathrm{mM}$ Tris buffer ( $\mathrm{pH} 8.8$ ), $0.1 \%(\mathrm{w} / \mathrm{v})$ SDS and included $0.1 \%$ bromophenol blue was added to the resolving gel. The IPG strips were placed onto the stacking gel and overlaid with $0.5 \%(\mathrm{w} / \mathrm{v})$ low melting agarose dissolved in $375 \mathrm{mM}$ Tris ( $\mathrm{pH} 8.8$ ): with $0.1 \%(\mathrm{w} / \mathrm{v})$ SDS. Electrophoresis was carried out at $4^{\circ} \mathrm{C}$; $150 \mathrm{~V}$ initially for $10 \mathrm{~min}$ then reduced to $90 \mathrm{~V}$ for $2.5 \mathrm{~h}$. The gels were placed in fixative containing $10 \%$ methanol and $7 \%$ acetic acid for 1 $\mathrm{hr}$. The gels were washed with distilled water for $20 \mathrm{~min}, 3$ times and subsequently stained with colloidal coomassie blue $(0.1 \%(\mathrm{w} / \mathrm{v})$ CCB G-250, 2\% (v/v) phosphoric acid, 10\% (w/v) ammonium sulphate, $20 \%$ (v/v) methanol) for $20 \mathrm{hr}$, with constant shaking at RT [14], the gels were then de-stained 5 times with $0.5 \mathrm{M} \mathrm{NaCl}, 15$ min each. Imaging of CBB-stained gels on the FLA-9000 imager (FUJIFILM, Tokyo, Japan) was carried out at $685 / 750$ excitation/emission with a photomultiplier tube (PMT) setting of $600 \mathrm{~V}$ and pixel resolution set to $100 \mu \mathrm{m}$ [14]. Analysis of 2D gel images was performed using Delta 2D software with automated spot detection (Local background region, 96; Average spot size, 32 and sensitivity in percentage, 20.0) (version 4.0.8; DECODON $\mathrm{GmbH}$, Greifswald, Germany). 
Citation: Stimpson SE, Lauto A, Coorssen JR, Myers SJ (2016) Isolation and Identification of ER Associated Proteins with Unique Expression Changes Specific to the V144D SPTLC1 Mutations in HSN-I. Biochem Anal Biochem 5: 248. doi:10.4172/2161-1009.1000248

Page 3 of 8

\section{Mass spectrometry}

2D gels were analysed for uniquely present or absent protein spots in control versus V144D mutant, (i.e. all or none changes) as previously described 25 and 18. Briefly, the protein species of interest were excised from gels and de-stained overnight. The gel pieces were then reduced and alkylated in $10 \mathrm{mM}$ Dithiothreitol (DTT) and $15 \mathrm{mM}$ Idoacetic acid (IAA), and subsequently incubated with trypsin solution (10 ng/ $\mu \mathrm{L}, \mathrm{pH}$ 7.4) for 16 hours at $37^{\circ} \mathrm{C}$. LC-MS/MS analysis was carried out on a nanoaquity UPLC (Waters Corp, Milford, MA, USA) linked to a Xevo QToF mass spectrometer from Waters (Micromass: UK). The data were acquired using Masslynx software (Version 4.1, Micromass UK). The MS/MS data files were searched against SwissProt databases with semi-trypsin as the enzyme.

\section{SDS-PAGE and immunoblotting}

Control and patient ER protein fractions $(25 \mu \mathrm{g}$ total protein) were subjected to SDS-PAGE on $12.5 \%$ resolving gels and transferred to PVDF membrane. The membranes were blocked with 5\% skim milk in TBS buffer containing $0.1 \%$ Tween-20. Whole membranes were blocked and incubated with anti-Calnexin (Cell Signalling Cat\# 2679S, RRID:AB_2228381), anti-SPTLC1 (Santa Cruz Biotechnology Cat\# sc-32916, RRID,AB_2195864), anti-Ig kappa chain C (Abcam Cat\# ab1050, RRID:AB_297240), anti-GAPDH (Abcam Cat\# ab9485, RRID:AB_307275), anti-ORP-150 (Abcam Cat\# ab124884, RRID:AB_10973544), anti-CLIC1 (Abcam Cat\# ab77214, RRID:AB_1566060), anti-RPS27a (Abcam Cat\# ab57646, RRID:AB_2180587) and anti-COTL1 (Proteintech Group Cat\# 107811-AP, RRID:AB_2084785) at 1:1000, for $16 \mathrm{~h}$. The membrane was then incubated with secondary HRP antibodies (Sigma-Aldrich Cat\# A9044, RRID:AB_258431 and Cat\# A0545, RRID:AB_257896) (1:2000 dilution) for $1 \mathrm{hr}$ at RT. Blots were developed using an enhanced chemiluminescence (ECL) detection kit (Pierce Thermo Scientific, USA). The membrane was developed on CL-Xposure Film (Thermo Fisher Scientific, U.S.A) using an AGFA X-ray developer.

\section{Immunofluorescence}

Immunofluorescence was carried out as previously described by Stimpson [16]. Briefly: Lymphoblasts $\left(1 \times 10^{6}\right.$ cells $)$ were suspended in $4 \%$ paraformaldehyde for $15 \mathrm{~min}$. Cells were then placed in $0.5 \%$ TritonX-100 and incubated at $37^{\circ} \mathrm{C}$ for $30 \mathrm{~min}$. The cells were then blocked in $5 \% \mathrm{BSA}$ solution at $37^{\circ} \mathrm{C}$ for $30 \mathrm{~min}$ : then resuspended in primary antibody, SPTLC1, ORP-150, CLIC1, RPS27a, COTL1, and stained for $1 \mathrm{hr}$ at RT. The cells were subsequently washed and resuspended in secondary antibody: anti-mouse Rhodamine (Millipore, 1:200), and incubated for 1 hour at RT. DAPI $(1 \mu \mathrm{g} / \mu \mathrm{L})$ was added to the cell suspension for $2 \mathrm{~min}$, the cells were centrifuged and washed twice with PBS. Aliquots $(300 \mu \mathrm{L})$ were added to 6 well culture plates containing coverslips coated in Histogrip (Invitrogen, USA) and centrifuged at $500 \times \mathrm{g}$ for $10 \mathrm{~min}$. The coverslips were washed in warm PBS, left overnight to dry and mounted onto glass slides prior to confocal imaging using the LSM 5 confocal microscope comprising the LSM 5 exciter laser scanning microscope with Axiovert $200 \mathrm{M}$ inverted optical microscope (Carl Zeiss: Jena: Germany).

\section{Flow cytometry}

FACS analysis was carried out as previously described by 18 . Lymphoblasts were isolated as above; the cells were then suspended in $4 \%$ paraformaldehyde and incubated for $15 \mathrm{~min}$ at RT and then resuspended in $0.3 \%$ Triton $\mathrm{X}-100$ for $15 \mathrm{~min}$ at $37^{\circ} \mathrm{C}$. After incubation the cell suspension was centrifuged at $1,000 \times \mathrm{g}$ for $5 \mathrm{~min}$ and the pellet resuspended in primary antibody for $1 \mathrm{hr}$ at RT. Cell suspension was centrifuged, washed in PBS and resuspended in secondary antibody, anti-mouse FITC (Millipore, 1:200) for $1 \mathrm{hr}$ at RT. The cell suspension was then analysed using the MACSQuant flow cytometer (Miltenyi Biotech).

\section{D gel images of ER proteins from control and patient de- rived lymphoblasts}

Total isolated ER proteins were resolved using a refined $2 \mathrm{DE}$ protocol $[13,14]$ (Figure 1). Control and V144D total ER proteins were resolved using mini gel format. Standard spot counts indicated $656 \pm 5$ and $675 \pm 3$ protein species were resolved in control and V144D mutant ER fractions respectively. Further analysis of the total ER protein profiles from control and HSN-I patient derived lymphoblasts revealed five 'all or none' protein changes in the V144D mutant and control lymphoblasts (Figure 2). These proteins were located in the $\mathrm{pI}$ of 5.5 , 5.6, 8.0, 5.8, 6.6 and 8.3 and molecular weight of 120,30, 15, 17, 22 and 22 respectively $(\mathrm{kDa})$ (Table 1$)$. Subsequent LC/MS analysis identified the protein to be ORP-150, CLIC1, RPS27a, Coactosin (COTL1) and Ig Kappa Chain C.

\section{Expression of identified ER proteins from HSN-I patient-de- rived lymphoblasts}

In order to determine protein expression changes, immunoblot analysis was carried out on isolated ER and total cell lysates from control and HSN-I patient derived lymphoblasts. These data showed a
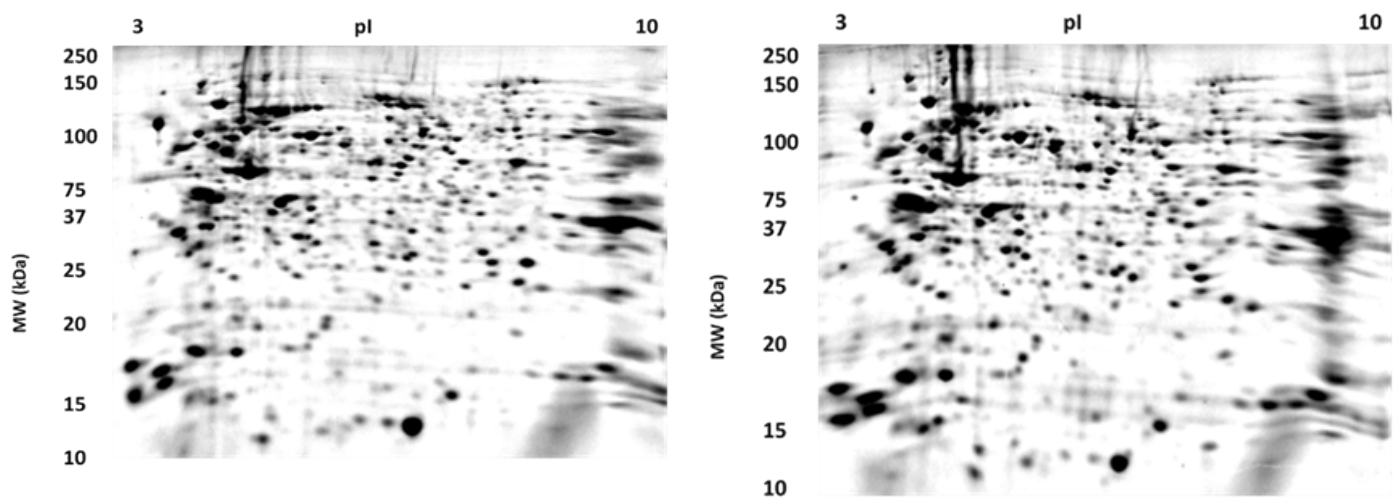

Figure 1: Representative images of 2D gels following resolution of ER proteins from control and patient derived lymphoblasts. (A) Control ER proteins; (B) V144D ER proteins. The molecular weights are in kilodaltons $(\mathrm{kDa})$ and the IEF dimension is in $\mathrm{pH}$ units 

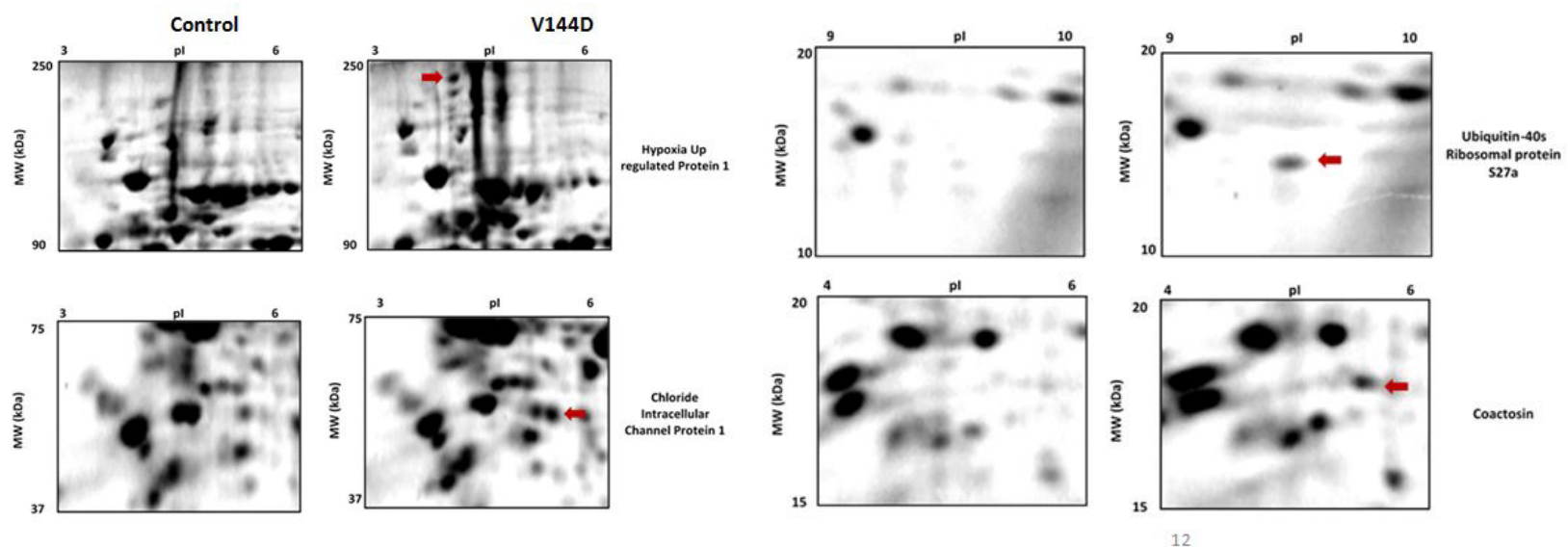

Control

V144D
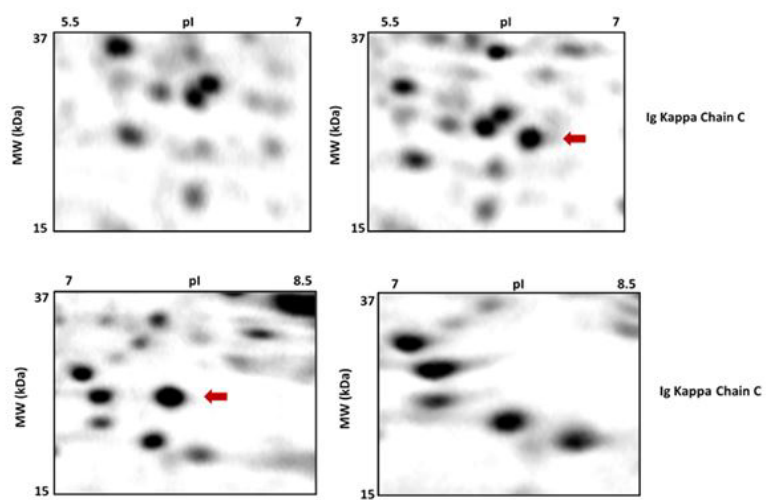

Figure 2: Representative images of 2D gel regions of ER proteins from control and patient derived lymphoblasts. Resolved protein species having a vastly altered abundance is indicated (Red Arrow). The molecular weights are in kilodaltons ( $\mathrm{kDa}$ ) and the IEF dimension is in $\mathrm{pH}$ units.

\begin{tabular}{|c|c|c|c|c|c|c|c|c|c|}
\hline $\begin{array}{l}\text { Spot } \\
\text { Number }\end{array}$ & Protein Identified & $\begin{array}{l}\text { Accession } \\
\text { Number }\end{array}$ & $\begin{array}{c}\text { Number of } \\
\begin{array}{c}\text { Unique peptides } \\
\text { matched }\end{array}\end{array}$ & $\begin{array}{l}\text { Sequence } \\
\text { Coverage }\end{array}$ & $\begin{array}{c}\text { Mascot Protein } \\
\text { Score }\end{array}$ & Predicted pl & $\begin{array}{l}\text { Predicted Mw } \\
\text { (kDa) }\end{array}$ & Mascot pl & $\begin{array}{c}\text { Mascot Mw } \\
\text { (kDa) }\end{array}$ \\
\hline I. & $\begin{array}{c}\text { Hypoxia Up } \\
\text { Regulated Protein } 1\end{array}$ & QY94L1 & 42 & $49 \%$ & 1741 & 5.5 & 120 & 5.2 & 112 \\
\hline II. & $\begin{array}{c}\text { Chloride Intracellular } \\
\text { Channel Protein } 1\end{array}$ & O00299 & 15 & $50 \%$ & 448 & 5.6 & 30 & 5.1 & 27.3 \\
\hline III. & $\begin{array}{c}\text { Ubiquitin-40s } \\
\text { Ribosomal protein } \\
\text { S27a }\end{array}$ & P62979 & 10 & $33 \%$ & 182 & 8.0 & 15 & 9.7 & 18.3 \\
\hline IV. & Coactosin & Q14019 & 19 & $73 \%$ & 190 & 5.8 & 17 & 5.6 & 16 \\
\hline V. & Ig Kappa Chain C & P01834 & 9 & $71 \%$ & 587 & 6.6 & 22 & 5.5 & 12 \\
\hline VI. & Ig Kappa Chain C & P01834 & 10 & $80 \%$ & 985 & 8.3 & 22 & 5.5 & 12 \\
\hline
\end{tabular}

Table 1: Summary table of mascot protein identification. LC-MS/MS and Mascot Database searching identified Hypoxia up Regulated Protein 1, Chloride Intracellula Channel Protein 1, Ubiquitin 40s ribosomal protein s27a, Coactosin and Ig Kappa Chain C from V144D patient derived lymphoblast control lymphoblasts isolated ER.

concomitant increase in the amount of ORP-150, CLIC1, and COTL1 in the V144D mutant protein samples compared to the control samples with a $p$ value of $<0.05$ (Figures 3A-3J). RPS27a was slightly increased in the V144D mutant; however this change was not statistically significant. Whereas quantitative analysis of Ig kappa protein displayed significant increase in the amount of Ig Kappa protein being expressed in the control total protein lysates compared to that of the protein isolated from the V144D mutant samples $(p<0.05)$ (Figure 3J).

The intracellular localisation and abundance of SPTLC1, ORP-150, CLIC1, RPS27a and COTL1 was established using immunofluorescence studies on control and patient-derived lymphoblasts. It was observed that there was no apparent change in intracellular localisation of the SPTLC1 protein in control and V144D mutant HSN-I patient lymphoblasts. However, whilst there appeared to be an increase in the abundance of ORP150, CLIC1, RPS27a and COTL1 in the V144D (patient) lymphoblasts, there was no change in intracellular localisation (Figure 4).

Control and patient-derived lymphoblasts were immuno stained for ORP-150, CLICL1, RPS27a and COTL1 were analysed by fluorescence assisted cell sorting (FACS) to determine the total fluorescence per cell (Figure 5). There was a marked increase in the relative fluorescence intensity of all the proteins in the V144D cells compared to that of control lymphoblasts with an increase in relative fluorescence of $1.4,1.25,2.4,1.5 \mathrm{OD}$ (fold increase) respectively, relative to the stained controls. 
Citation: Stimpson SE, Lauto A, Coorssen JR, Myers SJ (2016) Isolation and Identification of ER Associated Proteins with Unique Expression Changes Specific to the V144D SPTLC1 Mutations in HSN-I. Biochem Anal Biochem 5: 248. doi:10.4172/2161-1009.1000248
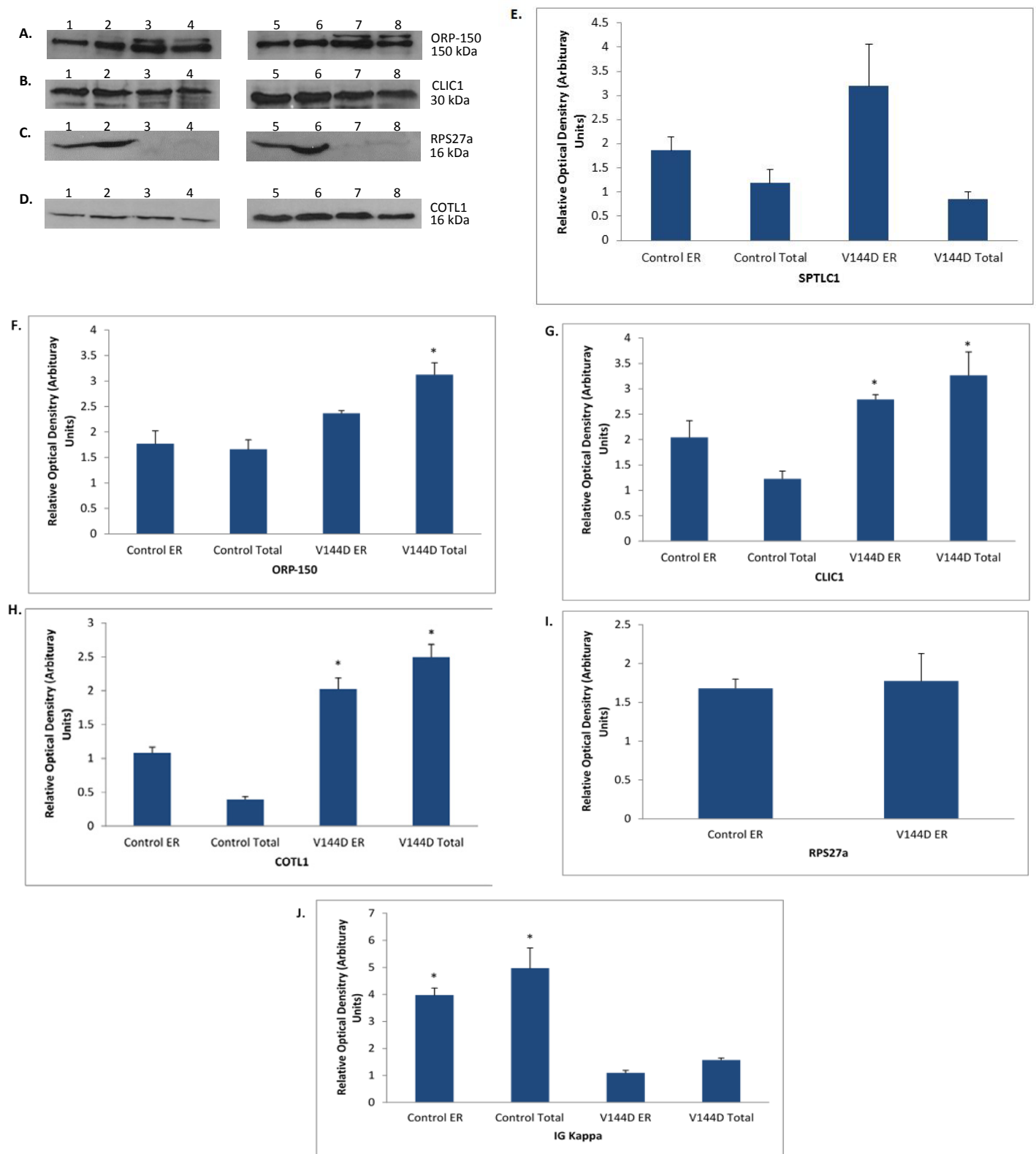

Figure 3: Expression of five ER proteins from HSN-I patient-derived lymphoblasts. (A) Immunoblot detection of ORP-150. (B) Immunoblot detection of CLIC1. (C) Immunoblot detection of RPS27a. (D) Immunoblot detection of COTL1. (E) Immunoblot detection of Ig Kappa Chain C. Lanes 1 and 2 represent control ER proteins, 3 and 4 represent control total proteins, 5 and 6 represent V144D ER proteins, 7 and 8 represent V144D total proteins. Figures (F-J) are representative graph showing statistical significant $\left.{ }^{*}\right)(p<0.05)$ difference between control patient lymphoblasts compared to the mutant V144D lymphoblasts of ORP-150, CLIC1, RPS27a, COTL1 and Ig Kappa Chain C respectively $(n=3)$. Blots normalised to GAPDH. Errors bar depict SE of means.
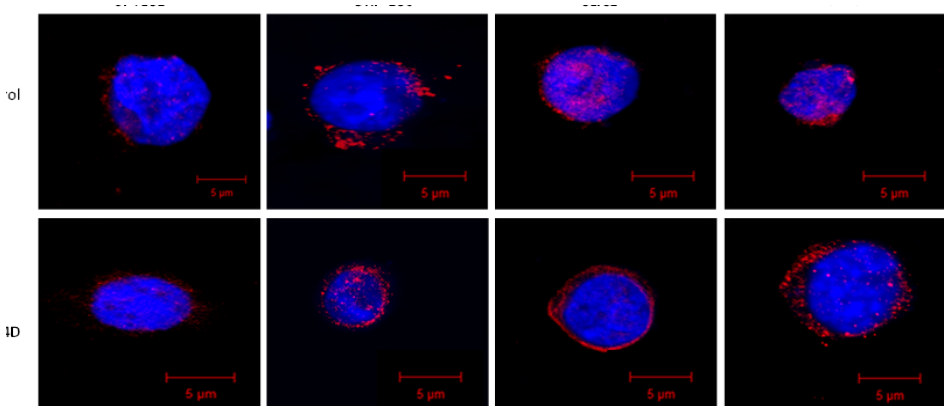
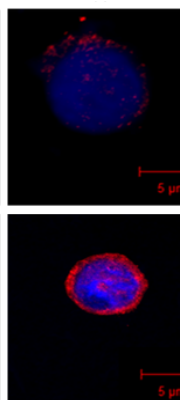

Figure 4: Representative immunofluorescence images of the intracellular localisation of five proteins. Representative confocal micrographs showing SPTLC1, CLIC1, ORP-150, RPS27a and COTL1 stained lymphoblasts (red) and DAPI nuclear stain (blue). Scale bar $=5 \mu \mathrm{m}$. 

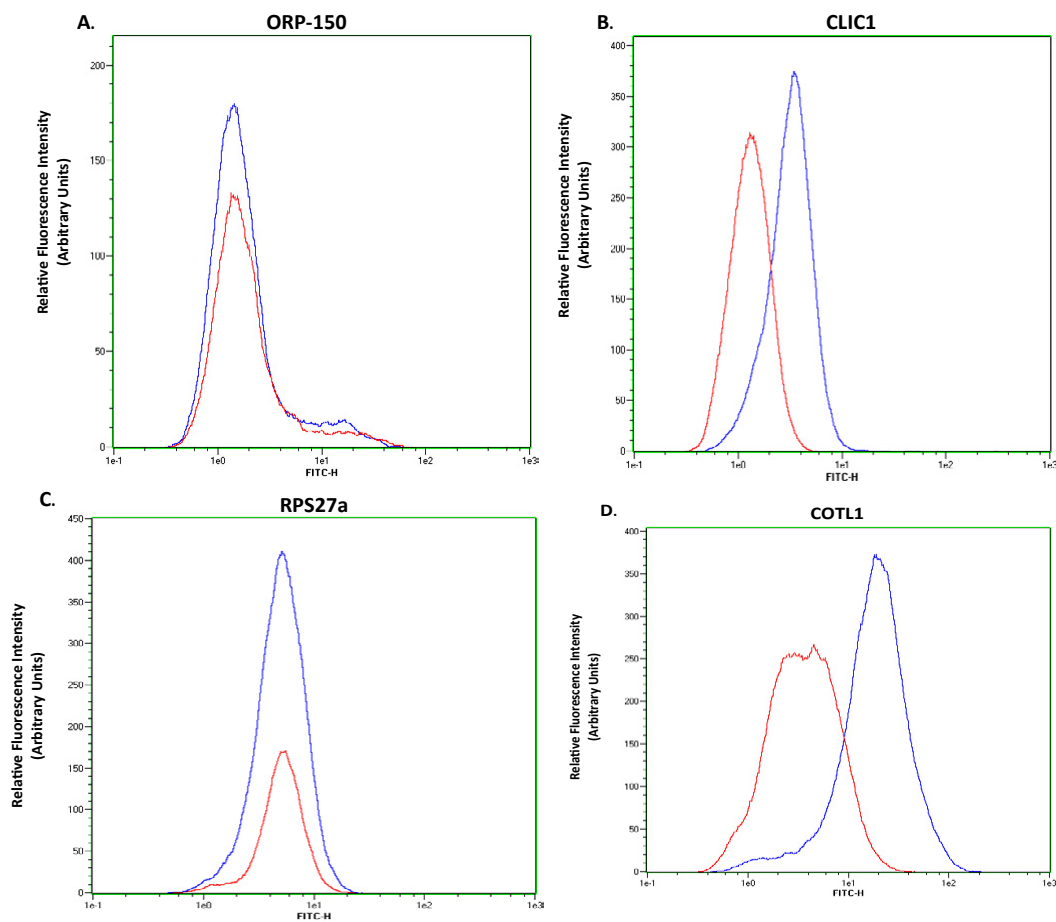

Figure 5: Relative quantification of ORP-150, CLICL1, RPS27a and COTL1 HSN-I patient-derived lymphoblasts expressing the V144D mutant SPTLC1 genes. Flow cytometry analysis of the relative fluorescence intensity of (A) ORP-150, (B) CLICL1, (C) RPS27a and (D) COTL1 in control and V144D patient-derived lymphoblasts. (A) Blue histogram represents the V144D patient lymphoblasts and Red histogram represents control lymphoblasts. ( $n=3)$.

\section{Discussion}

SPT is an ER bound and key rate determining enzyme in sphingolipid metabolism. Mutations within the SPT subunits result in potential dysfunction with possible perturbations in sphingolipid synthesis and metabolism causing HSN-I [2]. The ER plays a crucial role in many aspects of protein compartmentalisation which include membrane translocation, protein folding, post-translational modifications of proteins, transport of both membrane and soluble proteins, as well as monitoring protein synthesis and degradation [1]. These processes are both assisted and monitored by molecular chaperones. This investigation has identified several proteins that change in expression in the V144D SPTLC1 mutant lymphoblasts. Whilst this investigation only analysed one patient derived lymphoblast sample: we have conducted further investigation using a tranfected neuronal model, data in follow-up manuscript, with the data here correlating with the transfect neuronal system, thus indicating the changes observed here are not due to patient-patient variations. The proteins that have major increases in expression are ORP-150, CLIC1, COTL1, Ig Kappa Chain $\mathrm{C}$, and with an increase in RPS27a in the ER.

To elucidate if protein changes in the proteomes of the ER fractions occur due to mutations in SPTLC1 causing HSN-I ER membranes were isolated from control and V144D patient lymphoblasts and lysed proteins were subjected to high resolution 2DE (Figure 1). Calnexin, a marker for the ER: confirmed the quality of the isolated fraction used for analysis. In a study by 14, ER stress markers increased in V144D lymphoblasts: here we detected no statistically significant changes in expression of calnexin, suggesting that this protein is constant in both the control and V144D cells (Figures 6A-6F) Expression of the SPTLC1 protein in the $\mathrm{V} 144 \mathrm{D}$ fractions analysed, in comparison to the control and revealed no significant increase in expression (Figure 6E) indicating a constant expression state of SPTLC1 in the diseased state. GAPDH (a house keeping protein) analysis showed no significant changes in in the V144D compared to that of the control lymphoblasts (Figure 6F).

2DE analysis revealed 5 proteins species only detected in the V144D ER fractions in the pI of 5.5, 5.6, 8.0, 5.8, 6.6 and molecular weight of $120,30,15,17,24$ respectively (kDa) (Figure 2). Subsequent LC/MS analysis identified these protein species to be ORP-150, CLIC1, RPS27a, COTL1, and Ig Kappa Chain C (Table 1). A protein species was detected in the control ER fractions in the pI region of 8.3 and molecular weight range of $24 \mathrm{kDa}$. This protein species was identified as Ig Kappa Chain C. Quantitative immunoblot analysis was carried out to determine the expression of these 5 proteins: it was shown that ORP-150, CLIC1 and COTL1 had statistically significant increases in the V144D mutant (Figures 3A-3J), however RPS27a showed slight increase in the V144D mutant ER fraction: but was not statistically significant (Figure 3I).

Oxygen regulated proteins are overexpressed under conditions of hypoxia. The heat shock protein, oxygen-regulated protein of $150 \mathrm{kDa}$ (ORP-150) also known as Glucose related protein 170 (Grp170), serves as an important molecular chaperone of the endoplasmic reticulum during stress [17]. Notably, hypoxia mediated up-regulation of ORP150 suppresses programmed cell death driven by oxygen deprivation [18]. Neurons with increased ORP-150 expression demonstrated suppressed caspase-3-like activity [19].

Chloride intracellular channel protein 1 (CLIC1), is small in size and exists in both soluble cytoplasmic and integral membrane forms [20]. CLIC1 exists usually in a soluble form in the cytoplasm and nucleoplasm, but following stimuli undergoes major structural changes and inserts in lipid membranes, where it acts as a chloride-selective ion channel. Cell oxidation seems to be the most important stimulus controlling the transition of CLIC1 between these two forms [21]. 

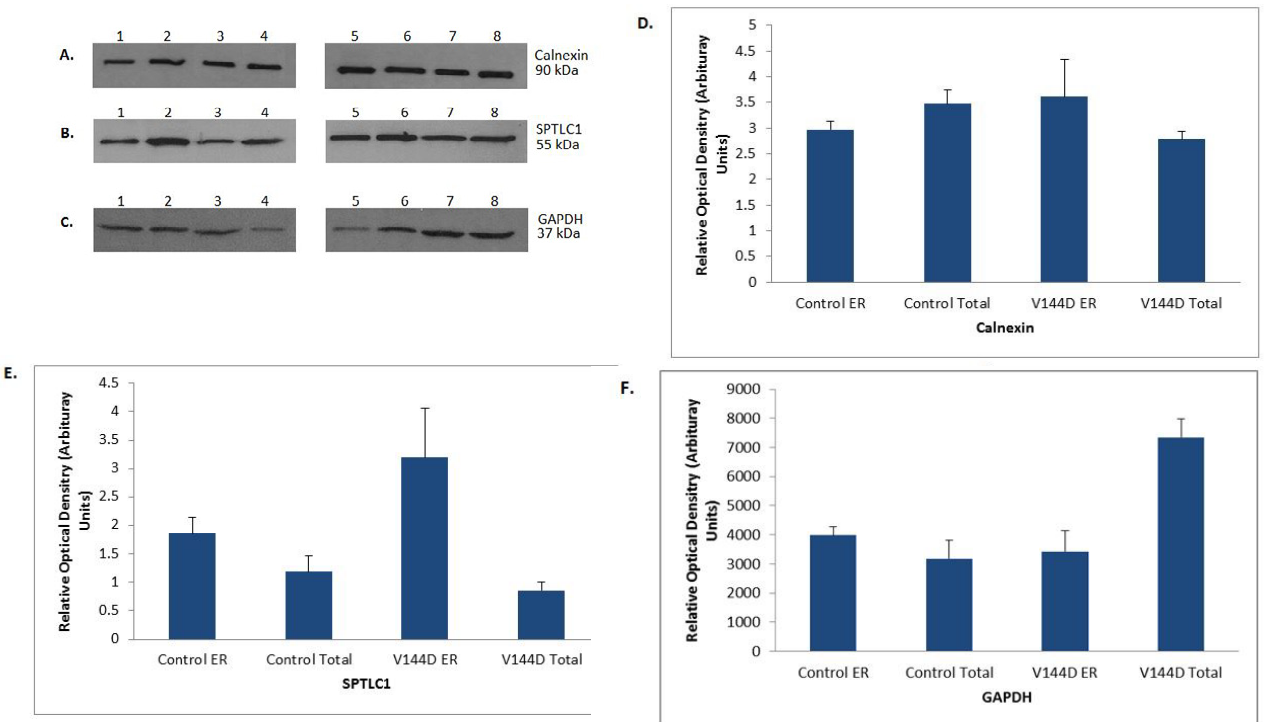

Figure 6: Expression of ER resident proteins and GAPDH in HSN-I patient-derived lymphoblasts. (A) Immunoblot of Calnexin, (B) Immunoblot of SPTLC1, (C) Immunoblot of GAPDH. Lanes 1 and 2 represent control ER proteins, 3 and 4 represent control total proteins, 5 and 6 represent V144D ER proteins, 7 and 8 represent V144D total proteins. A representative graph showing no statistical significant $(p>0.05)$ difference between ER control and patient lymphoblasts lysates $(n=3)$ of Calnexin $(D)$, SPTLC1 (E) and GAPDH (F). Blots were normalised to GAPDH. Errors bar depict SE of means.

While many of the processes that require ubiquitin: are common to all cell types, ubiquitin also has distinct roles in protein degradation. For example; the ubiquitin proteasome system: protein ubiquitylation is also responsible for regulating cell signalling by controlling the endocytosis of plasma membrane receptors. Ubiquitin: is highly conserved and is involved in processes of signal transduction, endocytosis, and DNA repair [22].

Cells are able to move and extend dynamically which is facilitated by actin dynamics. Coactosin (COTL1) is an actin binding protein, and has been shown to associate with F-actin [23]. Under normal cellular conditions, monomeric globular actin (G-actin) is in a state of equilibrium with filamentous actin (F-actin), forming the actin cytoskeleton and is responsible for maintaining and modifying cell shape in motility, phagocytosis, and cytokinesis [24]. The actin cytoskeleton is regulated by numerous actin-binding proteins that interact with actin and regulate the cytoskeleton in cells [23]. COTL1 was also found to directly interact with the filamentous, F-actin but does not form a stable complex with globular, G-actin [23].

Immunostaining (Figure 3) and FACS (Figure 4) analyses yielded the cellular localisation and expression of the following 5 proteins SPTLC1, ORP-150, CLIC1, RPS27a and COTL1 was established. It was observed that there was no apparent change in intracellular localisation of the SPTLC1 protein in control and V144D mutant HSN-I patient lymphoblasts. ORP-150, CLIC1, RPS27a and COTL1 displayed an intracellular localisation change to the cellular periphery and increased abundance. FACS analysis of ORP-150, CLIC1, RPS27a and COTL1 was used to determine the total fluorescence per cell, revealing a marked increase in the relative fluorescence intensity of all the proteins in the V144D cells compared to that of control lymphoblasts with an increase in relative fluorescence of 1.4, 1.25, 2.4, $1.5 \mathrm{OD}$ (fold increase) respectively: relative to the stained controls.

It was identified that there were two other proteins with a marked change in protein expression. Both proteins were located at $24 \mathrm{kDa}$, but each had a different pI ( 6.6 and 8.3 respectively). Following mass spectral analysis: both proteins were identified as Ig Kappa Chain C.
Ig kappa Chain $\mathrm{C}$ was found to significantly increase in the control lymphoblasts compared to that of the V144D mutant lymphoblasts (Figure 3J). This finding correlates with our previous studies in the V144D patient lymphoblasts [15].

\section{Conclusion}

The novel findings in this study thus suggest a link to increased oxidative stress within the V144D lymphoblasts. Previous studies $[8,15]$ have shown there is an increase in both ER stress and potential oxidative phosphorylation (via a potential change to ROS) changes in V144D. It is evident that there is an increase in oxidative stress within the V144D patient lymphoblasts demonstrated by the increased expression of ORP-150, CLIC1, COTL1 and RPS27a. While these proteins are functionally independent from one and another: together they help establish a strong connection that mutations in SPTLC1 cause oxidative stress within the cell. This increase in oxidative stress could be linked to the increase in Ubiquinol Cytochrome $\mathrm{C}$ expression from the mitochondria of V144D mutant cells previously observed: thus an increase in ORP-150 is observed to compensate and protect the cell from an increase in ROS production. Actin function is highly regulated by the association of actin binding proteins. Studies have shown that actin oxidation generally inhibits the association of actin binding proteins with actin [25]. As COTL1 is an actin binding partner its upregulation could be due to the increased oxidative stress upon the cellular cytoskeletal system. Oxidative stress can cause actin remodelling and potential axonal retraction in the neuron [22]. Under the conditions of stress the UPR is activated to ensure misfolded proteins are targets for destruction [22]. RPS27a has a major role in targeting cellular proteins for destruction as such its apparent increase in the V144D mutant demonstrates that there is a possible increase in misfolded protein either directly due to ER stress, oxidative stress or by another mechanism that affects protein conformation. The findings in this study, coupled with others $[8,15]$ suggest that there is a probable underlying mechanism that is common to sensory neurodegenerations.

\section{Acknowledgements}

We are grateful to Prof Garth Nicholson (Molecular Medicine Laboratory and 
Citation: Stimpson SE, Lauto A, Coorssen JR, Myers SJ (2016) Isolation and Identification of ER Associated Proteins with Unique Expression Changes Specific to the V144D SPTLC1 Mutations in HSN-I. Biochem Anal Biochem 5: 248. doi:10.4172/2161-1009.1000248

Page 8 of 8

Northcott Neuroscience Laboratory Anzac Research Institute: Sydney) for providing all EBV transformed lymphoblast lines used in this study. SS was supported by APA Research Scholarship: and the UWS School of Science and Health Postgraduate research fund. SM notes the continuing support of an anonymous Private Foundation. JC acknowledges the support of the UWS School of Medicine.

\section{Results}

\section{Expression of GAPDH and ER markers in HSN-I patient-de- rived lymphoblasts}

In order to assess the levels of calnexin and SPTLC1 expression in isolated ER fractions and total cell lysates from control and V144D mutant HSN-I patient-derived lymphoblasts: immunoblotting was carried out (Figures 6A-6F). Purity of the ER fraction was determined by immunoblotting for membrane, mitochondrial and Golgi complex markers: all of which were absent from the isolated ER fractions, indicating the isolated ER fraction was devoid of other organelles. There were no expression differences observed between the control and V144D samples in either isolated ER isolations or total cell lysates. Quantitation of the immunoblots of the isolated ER and total cell lysate fractions from control and HSN-I patient-derived lymphoblasts (Figures 6D and 6E): confirmed that there was no statistically significant change in expression of these proteins. Expression analysis of GAPDH (Figure 6C) established there was no statistically significant change in the expression of GAPDH (Figure 6F).

\section{References}

1. Pendin D, Mcnew JA, Daga A (2011) Balancing ER dynamics: shaping bending, severing, and mending membranes. Curr Opin Cell Biol 2: 435-442.

2. Wei J, Yerokun Y, Liepelt M, Momin A, Wang E, et al. (2007) 2-1 Serine Palmitoyltransferase. Sphingolipid Biology 25-27.

3. Verhoeven K, Timmerman V, Mauko B, Pieber TR, De Jonghe P, et al. (2006) Recent advances in hereditary sensory and autonomic neuropathies. Curr Opin Neurol 19: 474-480.

4. Dyck PJ, Thomas PK (2005) Dyck: Peripheral Neuropathy, 4th Edition, Mosby Elsevier, Philadelphia.

5. Lindholm D, Wootz H, Korhonen L (2006) ER stress and neurodegenerative diseases. Cell Death Differ 1: 385-392.

6. Rao RV, Bredesen DE (2004) Misfolded proteins, endoplasmic reticulum stress and neurodegeneration. Curr Opin Cell Biol 16: 653-662.

7. Fulda S, Gorman AM, Hori O, Samali A (2010) Cellular stress responses: cell survival and cell death. Int J Cell Biol 2010: 1-23.

8. Myers S, Malladi C, Hyland R, Bautista T, Boadle R, et al. (2014) Mutantions in the SPTLC1 protein cause mitochondrial structual abnormalisites and endoplasmic reticulum stress in lymphoblasts. DNA and Cell Biology 3: 7 .

9. Dedov V, Dedova I, Merrill A, Nicholson G (2004) Activity of partially inhibited serine palmitoyltransferase is sufficient for normal sphingolipid metabolism and viability of HSN1 patient cells. Biochimica et biophysica acta 1688: 168-175.

10. Bozidis P, Williamson CD, Colberg-Poley AM (2007) Isolation of endoplasmic reticulum, mitochondria, and mitochondria-associated membrane fractions from transfected cells and from human cytomegalovirus-infected primary fibroblasts. Curr Protoc Cell Biol Chapter 3: Unit 3: 27.

11. Vaseva AV, Moll UM (2013) Identification of p53 in mitochondria. Methods Mol Biol 962: 75-84.

12. Churchward MA, Butt RH, Lang JC, Hsu KK, Coorssen JR (2005) Enhanced detergent extraction for analysis of membrane proteomes by two-dimensional gel electrophoresis. Proteome Sci 3: 5.

13. Butt RH, Coorssen JR (2005) Postfractionation for enhanced proteomic analyses: routine electrophoretic methods increase the resolution of standard 2D-PAGE. J Proteome Res 4: 982-991.

14. Gauci VJ, Padula MP, Coorssen JR (2013) Coomassie blue staining for high sensitivity gel-based proteomics. J Proteomics 90: 96-106.

15. Stimpson S, Coorssen J, Myers S, (2014) Mitochondrial protein alterations in a familial peripheral neuropathy caused by the V144D amino acid mutation in the sphingolipid protein, SPTLC1. Journal of Chemical Biology 8: 25-35.

16. Wright EP, Partridge MA, Padula MP, Gauci VJ, Malladi CS, et al. (2014) Top down proteomics: enhancing $2 \mathrm{D}$ gel electrophoresis from tissue processing to high-sensitivity protein detection. Proteomics 14: 872-889.

17. Behnke J, Hendershot LM (2014) The large Hsp70 Grp170 binds to unfolded protein substrates in vivo with a regulation distinct from conventional Hsp70s. J Biol Chem 289: 2899-2907.

18. Stojadinovic A, Hooke JA, Shriver CD, Nissan A, Kovatich AJ, et al. (2007) HYOU1/Orp150 expression in breast cancer. Med Sci Monit 13: BR231-239.

19. Wu YB, Li HQ, Ren MS, Li WT, Lv XY, et al. (2013) CHOP/ORP150 ratio in endoplasmic reticulum stress: a new mechanism for diabetic peripheral neuropathy. Cell Physiol Biochem 32: 367-379.

20. Warton K, Tonini R, Fairlie WD, Matthews JM, Valenzuela SM, et al. (2002) Recombinant CLIC1 (NCC27) assembles in lipid bilayers via a pH-dependent two-state process to form chloride ion channels with identical characteristics to those observed in Chinese hamster ovary cells expressing CLIC1. J Biol Chem 277: 26003-26011.

21. Averaimo S, Milton RH, Duchen MR, Mazzanti M (2010) Chloride intracellula channel 1 (CLIC1): Sensor and effector during oxidative stress. FEBS Lett 584: 2076-2084.

22. Hallengren J, Chen PC, Wilson SM (2013) Neuronal ubiquitin homeostasis Cell Biochem Biophys 67: 67-73.

23. Provost P, Doucet J, StockA, Gerisch G, Samuelsson B, et al. (2001) Coactosinlike protein, a human F-actin-binding protein: critical role of lysine-75. Biochem J 359: 255-263.

24. Carlier MF, Laurent V, Santolini J, Melki R, Didry D, et al. (1997) Actin depolymerizing factor (ADF/cofilin) enhances the rate of filament turnover: implication in actin-based motility. J Cell Biol 136: 1307-1322.

25. Farah ME, Sirotkin V, Haarer B, Kakhniashvili D, Amberg DC (2011) Diverse protective roles of the actin cytoskeleton during oxidative stress. Cytoskeleton (Hoboken) 68: 340-354. 